Pacific Journal of Mathematics

THE 2-CELL AS A PARTIALLY ORDERED SPACE 


\section{THE 2-CELL AS A PARTIALLY ORDERED SPACE}

\section{E. D. TYMChATYN}

In this paper we prove a Jordan Curve Theorem (Theorem

1) for certain two dimensional partially ordered spaces.

We use this result to give a new characterization of the closed 2-cell (Theorm 2).

By a partially ordered space we $X$ mean a Hausdorff space $X$ with a partial order which is closed when regarded as a subset of $X \times X$ $(X \times X$ has the product topology).

For $x \in X$ we set

$$
\begin{aligned}
L(x) & =\{y \in X \mid y \leqq x\} \\
M(x) & =\{y \in X \mid x \leqq y\}
\end{aligned}
$$

and

$$
\Gamma(x)=L(x) \cup M(x)
$$

If $A \subset X$ we let

$$
L(A)=\bigcup\{L(x) \mid x \in A\} .
$$

We define $M(A)$ and $\Gamma(A)$ analogously. We let $L$ (resp. $M$ ) denote the set of minimal (resp. maximal) elements of $X$.

A chain is a totally ordered set. An order arc is a compact and connected chain. A separable and nondegenerate order arc is homeomorphic to $[0,1]$. A continuum is a compact, connected, Hausdorff space. An arc is a continuum with exactly two noncutpoints. A circle is a continuum such that every pair of points separates it.

Definition. If $X$ is a partially ordered space and $A \subset X$ let

$$
C(A)=L(A) \cap M(A) \text {. }
$$

A subset $A$ of $X$ is convex if and only if $A=C(A)$.

L. Nachbin proved the following result ([4], p. 48).

Lemma 1.1. (Nachbin). A compact partially ordered space $X$ has a basis of convex open sets.

The following three lemmas appear in [5]. For completeness we sketch their proofs here.

Lemma 1.2. Let $X$ be a compact partially ordered space such 
that $L$ is closed. If for each $x \in X L(x)$ has a unique minimal element $p(x)$ then the function $p: X \rightarrow L$ is a retraction.

Proof. We need only show $p$ is continuous. Let $\left(x_{i}\right.$ be a net converging to $x$ in $X$ and let $y$ be a cluster point of $\left.p\left(x_{i}\right)\right)$. Then $y \in L$ since $L$ is closed. Since the partial order on $X$ is closed $y \in L(x)$. Hence $y=p(x)$.

LEMma 1.3. Let $X$ be a compact partially ordered space such that $L$ is closed and for each $x \in X L(x)$ is an order arc. Let $2^{X}$ denote the space closed subsets of $X$ with the finite topology [3]. Then the function $f: X \rightarrow 2^{X}$ defined by $f(x)=L(x)$ is continuous.

Proof. It is well known (Michael [3]) that $2^{X}$ is a compact Hausdorff space and that the family of closed and connected subsets of $X$ is closed in $2^{X}$. Let $\left(x_{i}\right.$ be a net converging to $x$ in $X$ and let $A$ be a cluster point of $L\left(x_{i}\right)$ ). Since the partial order on $X$ is closed $A \subset L(x)$. Clearly $x \in A$ and $A$ meets $L$ since $L$ is compact. Since $A$ is connected and no proper connected subset of $L(x)$ contains both $x$ and $L(x) \cap L, A=L(x)$.

LEMMA 1.4. Let $X$ be a compact partially ordered space such that $L$ and $M$ are closed and for each $x \in X \Gamma(x)$ is an order arc. Then the projection $\pi: X \rightarrow M$ defined by letting $\pi(x) \in M(x) \cap M$ is continuous and open.

Proof. By Lemma 1.2 we need only show that $\pi$ is open. By Lemma 1.3 the function $f: M \rightarrow 2^{X}$ defined by letting $f(m)=L(m)$ is a homeomorphism onto $f(M) \subset 2^{X}$.

Let $x \in X$ and let $U$ be a neighborhood of $x$. Then the pair $\langle U, X\rangle$ is a basic open neighbourhood of $L(m)$ in $2^{X}$ (Michael [3]). Hence

$$
\pi(U)=f^{-1}(\langle U, X\rangle \cap f(M))
$$

is a neighbourhood of $\pi(x)$ in $M$.

Lemma 1.5. If $X$ is as in Lemma 1.4 then $X$ is locally connected if and only if $M$ is locally connected.

Proof. By Lemma $1.2 M$ is a retract of $X$ so $M$ is locally connected if $X$ is locally connected.

Suppose $M$ is locally connected and let $\pi$ be as in Lemma 1.4. Let $x \in X$ and let $U$ be a neighborhood of $x$. By Lemma 1.1 we may suppose $U$ is a convex open neighbourhood of $x$. By Lemma $1.4 \pi(U)$ 
is an open neighbourhood of $\pi(x)$ in $M$. Since $M$ is locally connected there exists a connected open set $V$ in $M$ such that $\pi(x) \in V \subset \pi(U)$. Then

$$
\pi^{-1}(V) \cap U=L(V) \cap U
$$

is a convex open neighbourhood of $x$. If $L(V) \cap U=A \cup B$ where $A$ and $B$ are nonvoid and open in $X$ then $\pi(A)$ and $\pi(B)$ are open in $M$ and $V=\pi(A) \cup \pi(B)$. Since $V$ is connected $\pi(A) \cap \pi(B)$ is nonvoid. Let $z \in \pi(A) \cap \pi(B)$. Then $L(z) \cap U=\pi^{-1}(z) \cap U$ is a connected set such that $L(z) \cap U \subset A \cup B$ and $L(z) \cap U$ meets both $A$ and $B$. Thus $A \cap B$ is nonvoid and $L(V) \cap U$ is connected.

Lemma 1.6. Let $X$ be a compact partially ordered space such that $L$ and $M$ are closed and for each $x \in X L(x)$ is an order arc. If $M$ is locally connected then $X$ is locally connected.

Proof. Define a set $Y$ by

$$
Y=\{(m, x) \mid m \in M \text { and } x \in L(m)\} .
$$

Give $Y$ the partial order $(m, x) \leqq{ }^{*}(n, y)$ if and only if $m=n$ and $x \in L(y)$. Define $g: Y \rightarrow X$ by $g(m, x)=x$ and give $Y$ the smallest topology $\mathscr{C}$ such that $g$ is continuous with respect to $\mathscr{C}$.

For each open set $V$ of $M$ let

$$
0_{V}=\{(m, x) \in Y \mid m \in V \text { and } x \in L(m)\} .
$$

Let $\mathscr{W}$ be the topology on $Y$ generated by $\mathscr{C}$ and

$$
\left\{0_{V} \mid V \text { is an open subset of } M\right\} \text {. }
$$

Then $\mathscr{W}$ is a Hausdorff topology. It follows from Alexander's Lemma (Kelly [7], p. 139) and Lemma 1.3 that $\mathscr{W}$ is a compact topology. Furthermore, the given partial order on $Y$ is closed with respect to $\mathscr{W}$. The detailed proofs of the above statements appear in [5], Theorem 2.7.

With the above partial order and the topology $\mathscr{W} Y$ is a compact partially ordered space which satisfies the hypotheses of Lemma 1.4. The set of maximal elements of $Y$ is homeomorphic to $M$. Hence, $Y$ is locally connected by Lemma 1.5. Now, $X$ is the continuous image of the compact, locally connected, Hausdorff space $Y$ so $X$ is locally connected.

Lemma 1.7. Let $X$ be a compact partially ordered space such that $M$ is a continuum and for each $x \in X L(x)$ is an order arc. If $F$ is a compact convex subset of $X$ such that for each $m \in M L(m) \cap F$ 
is nonvoid then $F$ is connected.

Proof. The relation $R$ on $F \times M$ defined by setting $(x, m) \in R$ if and only if $x \leqq m$ is upper-semicontinuous [2]. It follows by a wellknown result on upper-semicontinuous relations [2] that $F$ is connected.

2. A jordan curve theorem. In this section we shall prove the following theorem:

THEOREM 1. Let $X$ be a compact partially ordered space such that

(i) $M$ is an arc with endpoints 0 and 1 ,

(ii) $L$ is closed,

(iii) $L(m)$ is a nondegenerate order arc for each $m$ in $M$,

(iv) for each cutpoint $m$ of $M, L(m)$ separates $X$ into components $P$ and $Q$ such that either $\bar{P}$ or $\bar{Q}$ meets $L . \quad$ Let $B=L \cup M \cup L(0) \cup L(1)$. Then each circle in $X \backslash B$ separates $X$ and no pair of points separates $X$.

To prove Theorem 1 we shall use an approach somewhat similar to that used by Whyburn [6] in his proof of the Jordan Curve Theorem. We shall show that any circle in $X$ may be approximated arbitrarily closely by a circle which is the union of a finite number of convex arcs. We shall then prove that a circle which is the union of a finite number of convex arcs separates $X$.

For the remainder of this section let $X$ be as in Theorem 1. Let $M$ have its natural order $\leqq$ with initial point 0 . Then $a \leqq b$ in $M$ if and only if $a$ lies in every subcontinuum of $M$ which contains both 0 and $b$. For $a, b \in M$ with $a \leqq b$ let $[a, b]$ denote the arc in $M$ which is irreducible with respect to containing $a$ and $b$. Let

$$
[a, b[=[a, b] \backslash\{b\}
$$

and let

$$
] a, b]=[a, b] \backslash\{a\} .
$$

For $m \in M$ let

$$
P_{m}=L([0, m]) \backslash L(m)
$$

and let

$$
Q_{m}=L([m, 1]) \backslash L(m)
$$

Lemma 2.1. If $m \in M \backslash\{0,1\}$ then $P_{m}$ and $Q_{m}$ are connected and $P_{m}$ is separated from $Q_{m}$. 
LEMma 2.2. If $L$ is not trivial then $L$ is an arc.

Proof. By Lemma $1.2 L$ is a retract of $X$. Hence $L$ is connected. If $L$ is not a point then by condition (iv) of Theorem 1 the only noncutpoints of $L$ are in $L(0)$ and $L(1)$. Thus $L$ is an arc.

Lemma 2.3. If $x, z \in M$ with $x<z, y \in P_{z} \cap Q_{x}$ and $w \in L(x) \cap L(z)$ then $w \in L(y)$.

Proof. Let $m \in M \cap M(y)$. By Lemma $2.1 x<m<z$. Then $(L(z) \cup L(x)) \cap M(w)$ is a connected set which meets both components of $X \backslash L(m)$. Hence $w \in L(m)$. Now $y \in Q_{x}$ and $w \in L(x)$ so $y \geqq \equiv$. Since $L(m)$ is a chain and $y, w \in L(m) w \leqq y$.

Definition. An arc $C$ in $X$ is said to have $F T$ if $C$ is the union of a finite number of convex arcs. If $C$ is an arc with $F T$ then for each $m$ in $M L(m) \cap C$ consists of a finite number of components.

Definition. Let $C$ be an arc with $F T$ and let $x \in X \backslash C$. Let $D$ be a component of $C \cap L(x)$ such that $D$ does not contain an endpoint of $C$. We say $D$ is a turnabout of $C$ in $L(x)$ if and only if there exists a neighbourhood $U$ of $D$ in $C$ and $m \in M(x) \cap M$ such that $U \subset L([0, m])$ or $U \subset L([m, 1])$. If $D$ is a turnabout of $C$ in $L(x)$ then for each $n \in M(x) \cap M$ either $U \subset L([0, n])$ or $U \subset L([n, 1])$.

Lemma 2.4. Let $C$ be an arc with $F T$ and let $m \in M$ such that the endpoints of $C$ lie in $X \backslash L(m)$. The number of components of $C \cap L(m)$ which are not turnabouts of $C$ in $L(m)$ is odd if and only if exactly one of the endpoints of $C$ lies in $P_{m}$.

Proof. Let $A$ be a component of $C \cap L(m)$. Each sufficiently small neighbourhood of $A$ in $C$ meets both $P_{m}$ and $Q_{m}$ if and only if $A$ is not a turnabout of $C$. Hence the number of times that $C$ crosses $L(m)$ is odd if and only if the number of components of $C \cap L(m)$ which are not turnabouts of $C$ in $L(m)$ is odd.

Lemma 2.5. If $A$ is an arc in $X$ with endpoints $b$ and $c$ then there exists a convex arc $F$ with endpoints $b$ and $c$ such that $F \subset C(A)$.

Proof. If there exists $y \in A$ with $b, c \in M(y)$ let $x$ be maximal in $L(b) \cap L(c)$ and let

$$
F=M(x) \cap(L(b) \cup L(c)) .
$$

Then $F$ is a convex arc with endpoints $b$ and $c$ such that $F \subset C(A)$. 
Suppose, therefore, that there does not exist $y \in A$ with $b, c \in M(y)$. We may assume by Lemma 2.3 that if $m, n \in M$ with $b \in L(m)$ and $c \in L(n)$ then $m<n$. Let $r \in M(b) \cap M$ and let $s \in M(c) \cap M$ such that $r$ is maximal in $M(b) \cap M$ and $s$ is minimal in $M(c) \cap M$ (with respect to the total order on $M$ ). For each $x \in[r, s]$ let $g(x)$ be minimal in $L(x) \cap A$ and let

$$
G=\{g(x) \mid x \in[r, s]\} .
$$

For each $e \in[r, s]$ let $f_{e}$ be minimal in $[r, e]$ such that $g\left(f_{e}\right) \in L(e)$ and let $h_{e}$ be maximal in $[e, s]$ such that $g\left(h_{e}\right) \in L(e)$.

Let $e \in[r, s]$ such that $r<f_{e}$. Let $\left.e_{i}\right)_{i \in I}$ and $\left.d_{i}\right)_{i \in I}$ be two nets in $\left[r, f_{e}\left[\right.\right.$ which converge to $f_{e}$. Suppose the nets $\left.g\left(e_{i}\right)\right)$ and $g\left(d_{i}\right)$ ) converge to $m$ and $n$ respectively. Then $m, n \in L\left(f_{e}\right)$. Suppose $m<n$. By Lemma 1.1 there exist convex open neighbourhoods $U$ and $V$ of $m$ and $n$ respectively such that $L(U) \cap M(V)$ is void.

Pick $j \in I$ so that $D$, the arc in $A$ which is irreducible with respect to containing $g\left(e_{j}\right)$ and $m$, is contained in $U$. For each $i m \in Q_{d_{i}} \cap Q_{e_{i}}$. Also $g\left(e_{j}\right) \in P_{f_{e}} \cdot$ By Lemma 1.3 there exists $k \in I$ such that $g\left(e_{\jmath}\right) \in P_{d_{k}}$ and $g\left(d_{k}\right) \in V$. Then $L\left(d_{k}\right)$ separates $D$. Now

$$
L\left(d_{k}\right) \cap D \subset L\left(d_{k}\right) \cap A \cap U \neq \varnothing .
$$

If $z \in L\left(d_{k}\right) \cap A \cap U$ then $z<g\left(d_{k}\right)$ by the choice of $U$ and $V$. This contradicts the choice of $g\left(d_{k}\right)$. Hence $m=n$. We denote $m$ by $m_{e}$. If $t \in\left[f_{e}, h_{e}\right]$ then $g(t)=g(e)$ by Lemma 2.3. Similarly if $e \in[r, s]$ such that $h_{e}<s$ then

$$
\overline{g\left(\left[h_{e}, s\right]\right)} \cap L\left(h_{e}\right)
$$

consists of a single point. We denote this point by $n_{e}$.

If $e \in[r, s]$ such that $f_{e}=r$ we let $m_{e}=b$ and if $h_{e}=s$ we let $n_{e}=c$. For each $e \in[r, s]$ let $p_{e}$ be maximal in $L\left(m_{e}\right) \cap L\left(n_{e}\right)$ and let

$$
H=\left\{m_{e}, n_{e}, p_{e} \mid e \in[r, s]\right\} \text {. }
$$

Since $C(H) \subset C(G)$ it follows by the above argument that $H$ is closed.

We let $F=C(H)$. It is easy to check that $F$ is closed. By Lemma $1.7 F$ is connected. It is obvious from the above arguments that the only noncutpoints of $H$ are $b$ and $c$. Thus $F$ is a convex arc containing $b$ and $c$. Also $F \subset C(A)$.

Lemma 2.6. Let $A$ be an arc in $X$ with endpoints $b$ and $c$ and let $U$ be any neighbourhood of $A$. There exists an arc $E$ with $F T$ such that $E \subset U$ and the endpoints of $E$ are $b$ and $c$.

Proof. For each $x \in A$ let $V(x)$ be a closed and connected neighbour- 
hood of $x$ in $A$ such that $C(V(x)) \subset U$. Since $A$ is compact there exists an integer $n$ and $a_{1}, \cdots, a_{n} \in A$ with

$$
A \subset \bigcup\left\{V\left(a_{i}\right) \mid i=1, \cdots, n\right\} .
$$

We may suppose $n$ is the smallest such integer and that $V\left(a_{i}\right) \cap V\left(a_{j}\right)$ is nonvoid if and only if $|i-j| \leqq 1$.

The natural order on $A$ with initial point $b$ induces a total order on $V\left(a_{i}\right)$ for each $i=1, \cdots, n$.

By Lemma 2.5 there exists for each $i=1, \cdots, n$ a convex arc $B_{2 i-1}$ with the same endpoints as $V\left(a_{i}\right)$ such that

$$
B_{2 i-1} \subset C\left(V\left(a_{i}\right)\right) \subset U .
$$

For each $i=1, \cdots, n-1$ let $B_{2 i}$ be a convex arc whose initial point is the terminal point of $V\left(a_{i}\right)$ and whose terminal point is the initial point of $V\left(a_{i+1}\right)$ such that

$$
B_{2 i} \subset C\left(V\left(a_{i}\right)\right) \subset U .
$$

One can now construct by an induction argument an arc

$$
E \subset \bigcup\left\{B_{i} \mid i=1, \cdots, 2 n-1\right\} \subset U
$$

such that $E$ has $F T$ and the endpoints of $E$ are $b$ and $c$.

Lemma 2.7. Let $C$ be a convex arc in $X$ and let $m \in M \backslash C$ such that $L(m) \cap C$ is a turnabout of $C$ in $L(m)$. If $z$ is maximal in $C \cap L(m)$ then one of the components of $C \backslash z$ is a chain.

Proof. We may suppose that $C \subset L([0, m])$. Let $w$ be maximal in $M(z) \cap C$ and let $n$ be minimal in $M$ such that $w \in L(n)$. Then $n \in[0, m]$.

If $C \not \subset L([0, n])$ let $c \in C \backslash L([0, n])$. By Lemma 2.3

$$
L([0, m]) \subset L([0, n]) \cup M(z) .
$$

Hence $c \in M(z)$. Since $C$ is convex the component of $C \backslash z$ which contains $c$ lies in $M(z) \backslash(M(w) \backslash w)$. This component of $C \backslash z$ is a chain since $C$ is convex.

If $C \subset L(\mid 0, n])$ and $w$ is not an endpoint of $C$ let $F$ and $G$ be the components of $C \backslash w$. The endpoints of $C$ lie in $P_{n}$. Let $n_{i}$ ) be a net in [0, $n$ [ which converges to $n$. By Lemma $\left.1.3 L\left(n_{i}\right)\right)$ converges to $L(n)$ in $2^{x}$. Eventually, therefore, $L\left(n_{i}\right) \cap F$ and $L\left(n_{i}\right) \cap G$ are nonvoid. For each $i w \in Q_{n_{i}}$ hence

$$
L\left(n_{i}\right) \cap C=\left(L\left(n_{i}\right) \cap F\right) \cup\left(L\left(n_{i}\right) \cap G\right)
$$

is disconnected. This contradicts the assumption that $C$ is convex. 
Thus $w$ is an endpoint of $C$ and $(L(w) \cap M(z)) \backslash z$ is a component of $C \backslash z$ which is a chain.

LEMMA 2.8. Let $x \in X$ and let $U$ be a convex connected neighbourhood of $x$. Let $C$ be a convex arc in $X \backslash U$ such that $C$ has no endpoints in $L(U)$ and $C$ has a turnabout in $L(x)$. Then $L(U) \cap C$ is a chain and if $z \in U$ such that $L(z) \cap C$ is nonvoid then $L(z) \cap C$ is a turnabout of $C$ in $L(z)$.

Proof. Let $m \in M(x) \cap M$ and suppose $C \subset L([0, m])$. Let $y$ be maximal in $L(x) \cap C$. By Lemma 2.7 there is a component $T$ of $C \backslash y$ which is a chain. Then $T \subset M(y)$. Let $t$ be the endpoint of $C$ which is in $T$ and let $p \in M(t) \cap M$.

Let $z \in U \cap P_{m}$ and let $n \in M(z) \cap M$. Just suppose $p \in[n, m]$. Then $z \in P_{p}$ and so $L(p)$ separates $U$. Let $a \in L(p) \cap U$. Since

$$
L(t) \cap M(y) \subset C \text { and } C \cap U
$$

is void, $a \nsupseteq t$. Since $L(p)$ is a chain $t<a$. This contradicts the assumption that $C$ does not have an endpoint in $L(U)$. Thus $p<n$. By Lemma 2.3 it follows that $U \cap P_{m} \subset L(t)$. This proves the lemma.

Lemma 2.9. If $C$ is a circle with $F T$ in $X$ and $C \subset X \backslash M$, then $C$ separates $X$.

Proof. Let

$$
A=\left\{x \in X \backslash C \mid \begin{array}{l}
\text { the number of components of } C \cap L(x) \text { which } \\
\text { are not turnabouts of } C \text { in } L(x) \text { is odd }
\end{array}\right\}
$$

and let

$$
D=\left\{x \in X \backslash C \mid \begin{array}{l}
\text { the number of components of } C \cap L(x) \text { which } \\
\text { are not turnabouts of } C \text { in } L(x) \text { is even }
\end{array}\right\} .
$$

Then $X \backslash C=A \cup D$ and $A \cap D$ is void. We shall show first of all that $A$ and $D$ are open in $X$.

We may suppose that $C=A_{1} \cup \cdots \cup A_{q}$ where each $A_{i}$ is a convex arc such that if $A_{i} \cap A_{j}$ is nonvoid then either $A_{i}=A_{j}$ or $A_{i} \cap A_{j}$ consists of an endpoint of $A_{i}$ and $A_{j}$.

Let $x \in A$ and let $m \in M(x) \cap M$. Let $C_{1}, \cdots, C_{k}$ be the set of components of $C \cap L(x)$. By Lemmas 1.1, 1.3 and 1.6 there exists a convex connected neighbourhood $U$ of $x$ such that

(i) $U \subset X \backslash C$,

(ii) if $p \in L(U)$ is an endpoint of $A_{i}$ for some $i \in\{1, \cdots, q\}$ then $p \in L(x)$, 
(iii) if $i \in\{1, \cdots, q\}$ such that $A_{i}$ meets $L(U)$ then $A_{i}$ meets $L(x)$. We shall prove that $U \subset A$. For each $w \in U$ define a function $f_{w}$ with domain the set of components of $L(w) \cap C$ and with range the set of components of $L(x) \cap C$ as follows: Let $P$ be a component of $C \cap L(w)$. If $P$ meets $L(x)$ let $f_{w}(P)$ be the unique component of $C \cap L(x)$ which meets $P$. If $P$ does not meet $L(x)$ then $P \subset A_{i}$ for some unique $i \in\{1, \cdots, q\}$. Let $f_{w}(P)$ be the unique component of $C \cap L(x)$ which meets $A_{i}$. To prove that $U \subset A$ it will suffice to prove that for each $w \in U$ and each $i \in\{1, \cdots, k\}$ the number of elements of $f_{w}^{-1}\left(C_{i}\right)$ which are not turnabouts of $C$ in $L(w)$ is odd and only if $C_{i}$ is not a turnabout of $C$ in $L(x)$.

Let $y$ be maximal in $C_{1}$. We may suppose $A_{1}$ and $A_{2}$ each have exactly one endpoint in $C_{1}$ and that endpoint is $y$. We may also suppose $C_{1} \subset A_{1}$.

Case 1. Suppose $C_{1}$ is a turnabout of $C$ in $L(x)$. We may suppose $A_{1} \cup A_{2} \subset L([0, m])$.

Since $A_{1}$ has only one endpoint in $L(u)$ it follows that if $z \in U \cap P_{m}$ then $L(z)$ meets $A_{1}$.

Let $n$ be minimal in $M$ such that $y \in L(n)$ and let $n_{i}$ ) be a net in $\left[0, n\left[\right.\right.$ which converges to $n$. For each $i$ let $U_{i}=U \cap L\left(\left[0, n_{i}\right]\right)$. Since $L\left(n_{i}\right)$ separates $U$ and $U$ is convex and connected it follows that $U_{i}$ is connected. By the choice of $n$ and by Lemma 1.3

$$
U \backslash L([n, 1])=\bigcup U_{i} .
$$

Let $V=U \backslash L([n, 1])$ then $V$ is a convex connected open set such that $A_{1} \subset X \backslash V$ and the endpoints of $A_{1}$ lie in $X \backslash L(V)$. By Lemma 2.8 for each $z \in V L(z) \cap A_{1}$ is nonvoid and is not a turnabout of $A_{1}$ in $L(z)$. Similarly for each $x \in V L(z) \cap A_{2}$ is nonvoid and is not a turnabout of $A_{2}$ in $L(z)$.

If $z \in U \backslash V$ then $L(z) \cap\left(A_{1} \cup A_{2}\right)$ is either void or is a turnabout of $C$ in $L(z)$.

Case 2. Suppose $C_{1}$ is not a turnabout of $C$ in $L(x)$. We may suppose $A_{2} \subset L([0, m])$ and $A_{1} \subset L([m, 1])$.

If $z \in\left(U \cap P_{m}\right) \backslash M(y)$ then by the argument of Case $1 L(z) \cap A_{2}$ is nonvoid and is not a turnabout of $C$ in $L(z)$. Also $L(z) \cap A_{1} \subset L(y) \backslash\{y\}$. If $L(z) \cap A_{1}$ is nonvoid it is a turnabout of $C$ in $L(z)$.

If $z \in U \cap(L([m, 1]) \cup M(y))$ then $L(z) \cap\left(A_{1} \cup A_{2}\right)$ is nonvoid and connected and is not a turnabout of $C$ in $L(z)$.

Thus $U \subset A$ and $A$ is open. Similarly $D$ is open. Since $C$ is not an arc there exists $m \in M$ such that $C$ meets both $P_{m}$ and $Q_{m}$. By 
Lemma 2.4 there exists a component $E$ of $L(m) \cap C$, such that $E$ is not a turnabout of $C$ in $L(m)$. Let $x, y \in L(m) \backslash C$ such that

$$
E=M(x) \cap L(y) \cap C .
$$

If $x \in A$ then $y \in D$ and if $x \in D$ then $y \in A$. Thus both $A$ and $D$ are nonvoid and so $C$ separates $X$.

We are finally ready to prove Theorem 1.

Proof of Theorem 1. Let $C$ be a circle in $X \backslash B$ and let $m \in M$ such that $C$ meets both $P_{m}$ and $Q_{m}$. Let $a$ be maximal in $C \cap P_{m}$ and let $b$ be maximal in $Q_{m} \cap C$. Let $S$ and $T$ be the two $\operatorname{arcs}$ in $C$ which are irreducible with respect to containing $a$ and $b$.

Let $y$ be maximal in $C \cap L(m)$. We may suppose $y \in T$. Let $x$ be minimal in $T \cap L(m)$. Let $n \in L(x) \backslash C$ such that

$$
M(n) \cap L(x) \cap C=\{x\} .
$$

Suppose that $C$ does not separate $X$. Since $X \backslash C$ is connected and locally connected by Lemma 1.6 there exists a continuum $D$ in $X \backslash C$ such that $m, n \in D$.

Let $Z$ be the arc in $T$ which is irreducible with respect to containing $x$ and $y$. Let $U$ and $V$ be convex, open and connected neighbourhoods of $a$ and $b$ respectively such that the closure of $U \cup V$ does not meet $Z \cup L(m)$.

Let $Z^{\prime}, S^{\prime}$ and $T^{\prime}$ be arcs with $F T$ which are obtained from $Z, S$ and $T$ respectively by the method of Lemma 2.6 so that

$$
\begin{aligned}
& Z^{\prime} \subset X \backslash(D \cup S \cup U \cup V) \\
& S^{\prime} \subset X \backslash\left(D \cup Z^{\prime} \cup(L(x) \cap M(n))\right) \\
& T^{\prime} \subset X \backslash D
\end{aligned}
$$

and

$$
S^{\prime} \cap\left(T \cup T^{\prime}\right) \subset U \cup V
$$

Let $S^{\prime \prime}$ be an arc in $S^{\prime}$ which is irreducible with respect to having one endpoint in $T^{\prime} \cap U$ and the other in $T^{\prime} \cap V$. Let $T^{\prime \prime}$ be an arc in $T^{\prime}$ such that $E=S^{\prime \prime} \cup T^{\prime \prime}$ is a circle. Then $E$ is a circle with $F T$ in $X /(D \cup M)$.

Now, $T^{\prime \prime} \cap L(m) \subset L(y) \cap M(x)$ and the number of components of $T^{\prime \prime} \cap L(m)$ which are not turnabouts of $T^{\prime \prime}$ in $L(m)$ is odd by Lemma 2.4. Also,

$$
S^{\prime \prime} \cap L(m) \cap M(n) \subset(L(y) \backslash\{y\}) \cap(M(x) \backslash\{x\}) .
$$


Let $p, q \in Z^{\prime} \cap L(m)$ such that $p<q$ and

$$
M(p) \cap L(q) \cap Z^{\prime}=\{p, q\} .
$$

Let $R$ be the arc in $Z^{\prime}$ which is irreducible with respect to containing $p$ and $q$. Then

$$
\cdot P=R \cup(L(q) \cap M(p))
$$

is a circle with $F T$ in $X \backslash M$. Since $S^{\prime \prime} \cap Z^{\prime}$ is void

$$
P \cap S^{\prime \prime} \subset(L(q) \cap M(p)) \backslash\{p, q\} .
$$

The endpoints of $S^{\prime \prime}$ lie in the same component of $X \backslash P$ as does $m$. Hence, by Lemma 2.4 and Lemma 2.9 the number of components of $S^{\prime \prime} \cap L(q) \cap M(q)$ which are not turnabouts of $S^{\prime \prime}$ in $L(m)$ is even. It follows since $Z^{\prime}$ has $F T$ and $S^{\prime \prime} \cap Z^{\prime}$ is void that the number of components of $S^{\prime \prime} \cap L(m) \cap M(n)$ which are not turnabouts of $S^{\prime \prime}$ in $L(m)$ is even. Hence $m$ and $n$ lie in distinct components of $X \backslash E$. This is a contradiction since $E \cap D$ is void and $D$ is a continuum which contains $m$ and $n$. Thus $C$ separates $X$.

To prove that no pair of points separates $X$ it suffices to prove that if $m \in] 0,1]$ then $\bar{P}_{m} \cap L(m)$ is a nondegenerate arc. Let

$$
m \in] 0,1] \subset M \text { and let } p \in L(m) \backslash m
$$

such that $p \notin L(0)$. Let $n$ be minimal in $M$ such that $p \in L(n)$. Let $n_{i}$ ) be a net in [0, $n$ [ which converges to $n$. Br Lemma 1.3 the net $L\left(n_{i}\right)$ ) converges to $L(n)$. Hence $p \in \bar{P}_{n} \subset \bar{P}_{m}$.

3. Characterization of the 2-cell. We prove that if $X$ is as in Theorem 1 and also metric then $X$ is homeomorphic to the closed 2-cell.

THEOREM 2. If $X$ is a compact metric partially ordered space such that

(i) $M$ is an arc and $L$ is closed,

(ii) $L(m)$ is a nondegenerate order arc for each $m \in M$,

(iii) for each cutpoint $m$ of $M L(m)$ separates $X$ into components $P$ and $Q$ such that either $\bar{P}$ or $\bar{Q}$ meets $L$, then $X$ is homeomorphic to a closed 2-cell.

Proof. We shall use Bing's Characterization of the 2-sphere.

Clearly $X$ is a continuum. By Lemma $1.6 X$ is locally connnected. We proved in Theorem 1 that no pair of points separates $X$.

Let $D$ be the unit disc in the plane. Let $B$ be as in Theorem 1 . By Lemma 2.2, $B$ is a simple closed curve. Let $f: S^{1} \rightarrow B$ be a 
homeomorphism of the boundary $S^{1}$ of $D$ onto the subset $B$ of $X$. Let $Y$ be the adjunction space of $X$ with $D$ under the map $f$. We shall prove that $Y$ is a 2 -sphere. Since the boundary of $X$ in $Y$ is the simple closed curve $B$ it will follow that $X$ is a closed 2-cell.

It is clear that $Y$ is a locally connected, metric continuum such that no pair of points of $Y$ separates $Y$. It remains to show that every simple closed curve in $Y$ separates $Y$.

Let $C$ be a simple closed curve in $Y$. Let $y \in Y \backslash(X \cup C)$ and let $U$ be an open disc containing $y$ such that $\bar{U}$ is a closed disc in $Y \backslash X$. It is easy to define a closed partial order on $Y \backslash U$ so that $Y \backslash U$ satisfies all the hypotheses of Theorem 1 . Then $C$ is a simple closed curve in $Y \backslash U$ such that $C$ does not meet the boundary of $Y \backslash U$. By Theorem 1, $C$ separates $Y \backslash U$ and hence $C$ separates $Y$. Thus $Y$ is a 2-sphere and $X$ is a closed 2-cell.

\section{REFERENCES}

1. R. H. Bing, The Kline sphere characterization problem, Bull. Amer. Math. Soc. 52 (1946), 644-653.

2. S. P. Franklin Concerning continuous relations, UCLA dissertation, 1963.

3. E. Michael, Topologies on spaces of subsets, Trans. Amer. Math. Soc. 71 (1951), $152-182$.

4. L. Nachbin, Topology and order, Van Nostrand Company, Inc., New York, 1965.

5. E. D. Tymchatyn, Antichains and producis in partially ordered spaces (to appear)

6. G. T. Whyburn, Analytic topology, Amer. Math. Soc., New York, 1942.

7. J. L. Kelly, General topology, Van Nostrand Company, Inc., New York, 1955.

Received January 10, 1969. The research for this paper was supported in part by a grant from the National Science Foundation.

UNIVERSITY OF OREGON

EUGENE, OREGON

UNIVERSITY OF SASKatcheWAN

SASKatoon, SASKatcheWAN 


\section{PACIFIC JOURNAL OF MATHEMATICS}

\section{EDITORS}

H. ROYDEN

Stanford University

Stanford, California

\section{RichaRd PIERCE}

University of Washington Seattle, Washington 98105
J. DugundJI

Department of Mathematics

University of Southern California

Los Angeles, California 90007

BASIL GORDON

University of California

Los Angeles, California 90024

\section{ASSOCIATE EDITORS}
E. F. BECKENBACH
B. H. NeUMANN
F. WOLF
K. YOSHIDA

\section{SUPPORTING INSTITUTIONS}

UNIVERSITY OF BRITISH COLUMBIA CALIFORNIA INSTITUTE OF TECHNOLOGY

UNIVERSITY OF CALIFORNIA

MONTANA STATE UNIVERSITY

UNIVERSITY OF NEVADA

NEW MEXICO STATE UNIVERSITY

OREGON STATE UNIVERSITY

UNIVERSITY OF OREGON

OSAKA UNIVERSITY

UNIVERSITY OF SOUTHERN CALIFORNIA

\author{
STANFORD UNIVERSITY \\ UNIVERSITY OF TOKYO \\ UNIVERSITY OF UTAH \\ WASHINGTON STATE UNIVERSITY \\ UNIVERSITY OF WASHINGTON \\ AMERICAN MATHEMATICAL SOCIETY \\ CHEVRON RESEARCH CORPORATION \\ TRW SYSTEMS \\ NAVAL WEAPONS CENTER
}

The Supporting Institutions listed above contribute to the cost of publication of this Journal, but they are not owners or publishers and have no responsibility for its content or policies.

Mathematical papers intended for publication in the Pacific Journal of Mathematics should be in typed form or offset-reproduced, double spaced with large margins. Underline Greek letters in red, German in green, and script in blue. The first paragraph or two must be capable of being used separately as a synopsis of the entire paper. It should not contain references to the bibliography. Manuscripts, in duplicate if possible, may be sent to any one of the four editors. Please classify according to the scheme of Math. Rev. 36, 1539-1546. All other communications to the editors should be addressed to the managing editor, Richard Arens, University of California, Los Angeles, California, 90024.

50 reprints are provided free for each article; additional copies may be obtained at cost in multiples of 50 .

The Pacific Journal of Mathematics is published monthly. Effective with Volume 16 the price per volume (3 numbers) is $\$ 8.00$; single issues, $\$ 3.00$. Special price for current issues to individual faculty members of supporting institutions and to individual members of the American Mathematical Society: $\$ 4.00$ per volume; single issues $\$ 1.50$. Back numbers are available.

Subscriptions, orders for back numbers, and changes of address should be sent to Pacific Journal of Mathematics, 103 Highland Boulevard, Berkeley, California, 94708.

PUBLISHED BY PACIFIC JOURNAL OF MATHEMATICS, A NON-PROFIT CORPORATION

Printed at Kokusai Bunken Insatsusha (International Academic Printing Co., Ltd.), 7-17. Fujimi 2-chome, Chiyoda-ku, Tokyo, Japan. 


\section{Pacific Journal of Mathematics}

\section{Vol. 30, No. $3 \quad$ November, 1969}

Willard Ellis Baxter, Topological rings with property $(Y) \ldots \ldots \ldots \ldots . \ldots 5$

Sterling K. Berberian, Note on some spectral inequalities of $C . R$.

Putnam ..................................... 573

David Theodore Brown, Galois theory for Banach algebras . ........... 577

Dennis K. Burke and R. A. Stoltenberg, A note on p-spaces and Moore spaces ........................................ 601

Rafael Van Severen Chacon and Stephen Allan McGrath, Estimates of positive contractions....................................... 609

Rene Felix Dennemeyer, Conjugate surfaces for multiple integral problems in the calculus of variations ........................... 621

Edwin O. Elliott, Measures on countable product spaces.............. 639

John Moss Grover, Covering groups of groups of Lie type .............. 645

Charles Lemuel Hagopian, Concerning semi-local-connectedness and cutting in nonlocally connected continua .................. 657

Velmer B. Headley, A monotonicity principle for eigenvalues ........... 663

John Joseph Hutchinson, Intrinsic extensions of rings . . . . . . . . . . . . . 669

Harold H. Johnson, Determination of hyperbolicity by partial

prolongations .................................. 679

Tilla Weinstein, Holomorphic quadratic differentials on surfaces in $E^{3} \ldots 697$

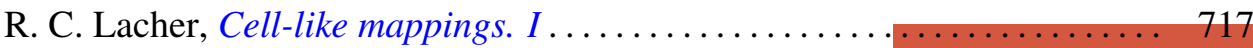

Roger McCann, A classification of centers

Curtis L. Outlaw, Mean value iteration of nonexpansive mappings in a

Banach space...

Allan C. Peterson, Distribution of zeros of solutions of a fourth order

differential equation.

Bhalchandra B. Phadke, Polyhedron inequality and strict convexity .. 765 Jack Wyndall Rogers Jr., On universal tree-like continua .

Edgar Andrews Rutter, Two characterizations of quasi-Frobenius rings

G. Sankaranarayanan and C. Suyambulingom, Some renewal theorems concerning a sequence of correlated random variables...

Joel E. Schneider, A note on the theory of primes........ . .

Richard Peter Stanley, Zero square rings .................

Edward D. Tymchatyn, The 2-cell as a partially ordered space

Craig A. Wood, On general Z.P.I.-rings................ 